Daniela Siqueira Prado'

Raquel Martins Arruda ${ }^{2}$

Raquel Cristina de Moraes Figueredo ${ }^{3}$

UMBERTO GAZI LIPPI ${ }^{4}$

manoel joão Batista Castello Girão

Marair Gracio Ferreira Sartori ${ }^{6}$

\section{Avaliação do impacto da correção cirúrgica de distopias genitais sobre a função sexual feminina}

Impact of surgery for pelvic organ prolapse on female sexual function
Palavras-chave

Prolapso uterino/cirurgia Vagina/cirurgia Soalho pélvico/patologia Comportamento sexual

Keywords

Uterine prolapse/surgery Vagina/surgery Pelvic floor/pathology Sexual behavior

Correspondência:

Daniela Siqueira Prado Rua Borges Lagoa, 933, apto. 91 - Vila Clementino CEP 04038-032 - São Paulo/SP Fone: (11) 5539-1908/9327-5900 E-mail:danisprado@ig.com.br

Recebido 02/07/2007

Aceito com modificacões $05 / 11 / 2007$

\section{Resumo}

OBJETIVO: avaliar as repercussões das cirurgias de correção de distopia genital sobre a função sexual feminina, bem como os resultados anatômicos pós-operatórios, e detectar possíveis correlações entre eles. MÉTODOS: estudo prospectivo realizado entre outubro de 2004 e setembro de 2006. Foram incluídas 43 mulheres sexualmente ativas com distopia genital com indicação de cirurgia de reconstrução do assoalho pélvico. No pré-operatório e três e seis meses após a cirurgia, as pacientes responderam ao questionário de avaliação do comportamento sexual e escalas analógicas para quantificação do grau de desejo, excitação e satisfação, além de se submeterem a exame físico para graduação da distopia genital. Para análise dos resultados, utilizaram-se os testes de simetria de Bowker, Wilcoxon, t de Student, $\chi^{2}$ e análise de variância (ANOVA), quando indicados, com limite de significância estatística de 5\% (p<0,05). RESULTADOS: as 43 mulheres completaram o seguimento de três e seis meses após a cirurgia, mas duas perderam os parceiros. Houve melhora significativa na qualificação da vida sexual ( $p=0,03)$. Dispareunia $(25,6 \%$ no pré-operatório versus $17,1 \%$ no pós-operatório), incômodo $(27,9$ versus $0 \%)$, embaraço $(20,9$ versus $0 \%)$ e medo $(2,3$ versus $0 \%)$ melhoraram de forma significativa $(p<0,001)$. As escalas analógicas de desejo $(5$ versus $7, p=0,001)$, excitação (6 versus $8, p<0,001)$ e satisfação com a vida sexual $(5$ versus $7, p<0,001)$ também apresentaram melhora significativa. Houve melhora significativa entre os estádios clínicos do pré-operatório e seis meses após a cirurgia $(p<0,001)$. Não houve correlação significativa entre as alterações nas dimensões vaginais e a mudança na função sexual. CONCLUSÕES: após cirurgias de reconstrução do assoalho pélvico, houve melhora significativa na qualificação da vida sexual e no estadiamento clínico das distopias. No entanto, não houve correlação entre estes indicadores.

\section{Abstract}

PURPOSE: to identify the impact of pelvic reconstructive surgery on female sexual function, as well as the changes in vaginal anatomy, and to detect possible correlations between them. METHODS: a prospective, descriptive study, including 43 sexually active women with genital dystopy, undergoing surgery for pelvic organ prolapse, conducted between October 2004 and September 2006. The women completed the same multiple-choice questionnaire regarding sexual function, and analogic scales to quantify the degree of desire, arousal and satisfaction, and were clinically assessed using the pelvic organ prolapse quantification (POP-Q) staging system, before the surgery and three and six months after it. Statistical analysis was performed through the Bowker test for symmetry, Wilcoxon test, Student $t$ test, $\chi^{2}$ and analysis of variance (ANOVA) as appropriate, with statistical significance set at $5 \%$ (p<0.05). RESULTS: all 43 women completed the follow-up at three and six months after the surgery, but two of them lost their partners after the surgery. Quality of sexual life improved significantly $(\mathrm{p}=0.03)$. Symptoms such as dyspareunia $(25.6 \%$ before versus $17.1 \%$ after surgery), discomfort $(27.9$ versus $0 \%)$, embarrassment $(20.9 \%$ versus $0 \%)$ and fear $(2.3 \%$ versus $0 \%)$ significantly improved $(p<0.001)$. Analogical scales scores regarding desire $(5$ versus $7, p=0.001)$, arousal $(6$ versus 8 , $\mathrm{p}<0.001)$ and satisfaction with sexual life (5 versus $7, \mathrm{p}<0.001)$ also improved. There was a statistically significant improvement $(p<0.001)$ of the POP-Q stages after the surgery. However, there was no statistically significant correlation between changes in vaginal dimensions and changes in sexual function. CONCLUSIONS: after pelvic reconstructive surgery, there was a significant improvement in the quality of sexual life and of the POP-Q stages. However, there was no correlation between them.

Trabalho realizado no Setor de Uroginecologia e Cirurgia Vaginal da Escola Paulista de Medicina da Universidade Federal de São Paulo - UNIFESP - São Paulo (SP), Brasil e do Hospital do Servidor Público Estadual de São Paulo "Francisco Morato de Oliveira" -HSPE-FMO - São Paulo (SP), Brasil.

'Pós-graduanda do Setor de Uroginecologia e Cirurgia Vaginal, Disciplina de Ginecologia Geral da Universidade Federal de São Paulo - UNIFESP - São Paulo (SP), Brasil; Médica do Serviço de Ginecologia e Obstetrícia do Hospital do Servidor Público Estadual de São Paulo "Francisco Morato de Oliveira" - HSPE-FMO - São Paulo (SP), Brasil.

${ }^{2}$ Médica do Setor de Uroginecologia e Cirurgia Vaginal da Universidade Federal de São Paulo - UNIFESP - São Paulo (SP), Brasil, e do Hospital do Servidor Público Estadual de São Paulo "Francisco Morato de Oliveira" -HSPE-FMO - São Paulo (SP), Brasil.

${ }^{3}$ Encarregada do Setor de Uroginecologia e Cirurgia Vaginal do Hospital do Servidor Público Estadual de São Paulo "Francisco Morato de Oliveira" - HSPE-FMO - São Paulo (SP), Brasil.

${ }^{4}$ Diretor do Serviço de Ginecologia e Obstetrícia do Hospital do Servidor Público Estadual de São Paulo "Francisco Morato de Oliveira" - HSPE-FMO - São Paulo (SP), Brasil.

5Professor Titular do Departamento de Ginecologia da Universidade Federal de São Paulo - UNIFESP - São Paulo (SP), Brasil.

- Professora Associada e Chefe da Disciplina de Ginecologia Geral da Universidade Federal de São Paulo - UNIFESP - São Paulo (SP), Brasil. 


\section{Introdução}

É crescente o interesse na avaliação das disfunções sexuais femininas, principalmente após a realização do National Health and Social Life Survey (NHSLS), que incluiu 1.749 mulheres e detectou prevalência de queixas sexuais em $43 \%$ destas ${ }^{1}$. No Estudo da Vida Sexual dos Brasileiros (EVSB), realizado entre novembro de 2002 e fevereiro de 2003, com 7.103 indivíduos de 13 Estados brasileiros, observou-se freqüência de disfunções sexuais de $48,1 \%$ para os homens e de $50,9 \%$ para as mulheres ${ }^{2}$.

As pacientes de clínicas ou ambulatórios de uroginecologia apresentam maior risco para disfunção sexual do que a população geral, por terem, freqüentemente, idade avançada (dificuldade de lubrificação e redução do desejo) e desordens do assoalho pélvico que geram incontinência urinária de esforço, bexiga hiperativa, incontinência fecal e distopia genital ${ }^{3,4}$. As mulheres com distopia genital podem ser assintomáticas ou ter dor hipogástrica, sintomas miccionais, intestinais ou disfunção sexual ${ }^{5-7}$.

A relação entre distopia genital e função sexual é pouco compreendida. Mulheres com distopia podem ter dispareunia, redução da capacidade para atingir o orgasmo, redução da libido, embaraço ou medo pela sua anatomia alterada ${ }^{5-8}$.

A interferência positiva ou negativa das cirurgias de reconstrução do assoalho pélvico na função sexual feminina é controversa ${ }^{9-11}$. Alguns investigadores demonstram resultados satisfatórios com melhora ou inalteração da função sexual e satisfação em $89 \%$ das pacientes após cirurgias para correção de distopia e/ou incontinência urinária de esforço ${ }^{10}$, enquanto estudo multicêntrico prospectivo refere redução na freqüência de intercurso, do desejo, da excitação, da obtenção de orgasmos, bem como da satisfação com a função sexual, após tais cirurgias ${ }^{11}$.

Além disso, alguns trabalhos não utilizam questionários validados ${ }^{9,10}$ e/ou não procedem à graduação da distopia conforme recomenda a Sociedade Internacional de Continência (ICS) ${ }^{12}$ no pré-operatório e nos retornos após a cirurgia ${ }^{9,11,13}$.

Tendo em vista a escassez de trabalhos prospectivos e que tenham utilizado a graduação da distopia genital conforme recomendação da ICS ${ }^{12}$, especialmente em nosso meio, interessou-nos avaliar as repercussões das cirurgias para correção de distopia genital sobre a função sexual feminina, bem como os resultados anatômicos pósoperatórios, e detectar possíveis correlações entre eles.

\section{Métodos}

Foi realizado estudo prospectivo, descritivo, no Setor de Uroginecologia e Cirurgia Vaginal da Disciplina de
Ginecologia Geral do Departamento de Ginecologia da Escola Paulista de Medicina da Universidade Federal de São Paulo (UNIFESP) e do Hospital do Servidor Público Estadual de São Paulo "Francisco Morato de Oliveira”. Foram selecionadas 43 mulheres no período de outubro de 2004 a setembro de 2006, após a aprovação do projeto pelo Comitê de Ética em Pesquisa de ambas as instituições.

Foram incluídas pacientes com distopia genital com indicação de cirurgia de reconstrução do assoalho pélvico, sexualmente ativas. Os critérios de exclusão foram: presença de queixas importantes de perda involuntária de urina, caracterizada por pressão de perda inferior a $90 \mathrm{cmH}_{2} \mathrm{O}$, ou relato de perda urinária durante o intercurso e inatividade sexual há mais de um ano.

A histerectomia vaginal foi realizada em todas as pacientes. Correção de cistocele e perineoplastia foram associadas à histerectomia vaginal em $84 \%$ dos casos, colpofixação no ligamento sacro-espinhoso em 1,4\% e correção de incontinência urinária de esforço leve com sling de polipropileno via transobturadora $\left(\right.$ TVT-O $\left.^{\circledR}\right)$ em $21 \%$. As cirurgias foram feitas por médicos residentes do terceiro ano ou por pós-graduandos em uroginecologia, sob supervisão e auxílio de médicos assistentes.

Após assinatura do termo de consentimento pósinformado, no pré-operatório e três e seis meses após a cirurgia, as pacientes responderam ao questionário de avaliação do comportamento sexual validado no Brasil ${ }^{14}$ e escalas analógicas para quantificação do grau de desejo, excitação e satisfação com sua vida sexual. Apenas uma paciente com os critérios de inclusão se negou a participar do estudo. O questionário consta de quatro grupos de questões: de identificação, de saúde geral, de hábitos e de práticas sexuais, fornecendo ampla noção do perfil sexual das pacientes. Para a avaliação do impacto das cirurgias na função sexual feminina, foram analisados alguns itens em particular: a freqüência de atividade sexual, a satisfação com as preliminares, a excitação, a qualificação da vida sexual, a existência de queixas e a freqüência de orgasmos.

Todas submeteram-se a exame físico, pelo mesmo investigador que aplicou o questionário, para graduação da distopia genital segundo sistema recomendado pela ICS ${ }^{12}$ também no pré-operatório e três e seis meses após a cirurgia.

Para a análise estatística das respostas e achados de exame físico no pré-operatório e seis meses após a cirurgia, utilizou-se o teste de simetria de Bowker (generalização do teste de McNemar) para variáveis categóricas com mais de duas categorias, como satisfação com as preliminares, freqüência de obtenção de orgasmos, qualificação da vida sexual e queixas referidas pelas pacientes. As 
respostas à variável "freqüência de atividade sexual" foram comparadas pelo teste $t$ de Student pareado. A comparação dos escores de desejo, excitação e satisfação foi realizada pelo teste não paramétrico de Wilcoxon para amostras dependentes. Para outras comparações de variáveis categóricas (associação entre impacto das cirurgias e estádio clínico), utilizou-se o $\chi^{2}$, e análise de variância (ANOVA) para variáveis numéricas como hiato genital e comprimento total da vagina, quando associados ao impacto da cirurgia.

O nível de significância adotado foi de $5 \%(\alpha=0,05)$ e níveis descritivos (p) inferiores a esse valor foram considerados significantes.

\section{Resultados}

A média de idade das 43 componentes do estudo foi de 52,9 anos. A maioria das pacientes era casada (79\%), católica $(69,8 \%)$ e branca $(65,1 \%)$. O início da atividade sexual foi, em média, com 20,6 anos. A paridade, em média, foi de 3,3 partos e $53,5 \%$ das pacientes encontravam-se na pós-menopausa.

Todas as mulheres incluídas no estudo retornaram para reavaliação após três e seis meses da cirurgia. No entanto, nove ainda não tinham tido atividade sexual no terceiro mês após a cirurgia e duas, no sexto mês, por perda do parceiro. Desse modo, a avaliação estatística da função sexual pós-operatória foi realizada com os resultados referidos no sexto mês após a cirurgia.

Não foi verificada diferença significativa $(p=0,16)$ entre a freqüência de atividade sexual no pré-operatório $(5,1)$ e seis meses após a cirurgia $(5,6)$.

Quando questionadas a respeito das preliminares, $69,7 \%$ das pacientes referiam estar satisfeitas ou muito satisfeitas no pré-operatório e $63,4 \%$, seis meses após a cirurgia. Tal variação não foi significativa $(\mathrm{p}=0,98)$.

Todas as pacientes referiam ter excitação, apesar de $67,4 \%$ demorarem a ficar excitadas no pré-operatório, $50 \%$ com três meses de pós-operatório e 53,6\% após seis meses de cirurgia.

Tabela 1 - Qualificação da vida sexual no pré-operatório, três e seis meses após a cirurgia.

\begin{tabular}{|c|c|c|c|c|}
\hline Vida sexual & $\begin{array}{c}\text { Pré-operatório } \\
\text { n (\%) }\end{array}$ & $\begin{array}{c}3 \text { meses } \\
\text { n (\%) }\end{array}$ & $\begin{array}{c}6 \text { meses } \\
n(\%)\end{array}$ & $\underset{\text { (início/fim) }}{p}$ \\
\hline Muito ruim & $3(7,0)$ & $1(2,9)$ & $1(2,4)$ & \\
\hline Ruim & $8(18,6)$ & $3(8,8)$ & $4(9,8)$ & \\
\hline Regular & $15(34,9)$ & $10(29,4)$ & $9(22,0)$ & $0,03^{*}$ \\
\hline Boa & $16(37,2)$ & $16(47,1)$ & $23(56,0)$ & \\
\hline Ótima & $1(2,3)$ & $4(11,8)$ & $4(9,8)$ & \\
\hline Total & $43(100)$ & $34(100 \%)$ & 41 (100) & \\
\hline
\end{tabular}

*Valor do p obtido pelo teste de simetria de Bowker.
A freqüência de obtenção de orgasmos se manteve inalterada em $34,1 \%$ dos casos, piorou em $17,1 \%$ e melhorou em $48,8 \%$, mas tais alterações não foram também significativas $(\mathrm{p}=0,15)$.

Quando interrogadas a respeito da qualidade da vida sexual (muito ruim, ruim, regular, boa ou ótima), observou-se que aproximadamente $46 \%$ das pacientes tiveram melhores respostas (boa/ótima) após a cirurgia. Deste modo, houve melhora significativa $(\mathrm{p}=0,03)$ na qualificação da vida sexual (Tabela 1).

A dispareunia, queixa presente em $25,6 \%$ das pacientes no pré-operatório, foi citada por $17,1 \%$ seis meses após a cirurgia. Das sete pacientes que referiram tal sintoma no pós-operatório, cinco já o apresentavam no pré-operatório. Das que não referiam tal sintoma antes da cirurgia, em uma, o sintoma apareceu como conseqüência de fibrose perineal acentuada, e, na outra, pela presença de abscesso pélvico (única complicação pós-operatória demonstrada neste estudo). Incômodo $(27,9 \%)$, embaraço $(20,9 \%)$ e medo $(2,3 \%)$ referidos no pré-operatório não foram detectados após a cirurgia (Tabela 2).

Os escores obtidos com as escalas analógicas apresentaram melhora significativa. $\mathrm{O}$ desejo variou de 5 para $7(\mathrm{p}=0,001)$, a excitação, de 6 para $8(\mathrm{p}<0,001)$ e a satisfação, de 5 para $7(\mathrm{p}<0,001)$, conforme Tabela 3 .

O impacto das cirurgias de correção de distopia genital sobre a função sexual feminina foi positivo (melhor) para 70,7\% das pacientes, negativo (piora) para $12,2 \%$ e não houve alteração para $17,1 \%$.

Todas as pacientes apresentavam distopia genital com estádio mínimo de II no pré-operatório. Foi observada melhora significativa $(\mathrm{p}<0,001)$ entre os estádios no pré-operatório e seis meses após a cirurgia (em 46,6\% houve redução do estadiamento), como demonstra a Tabela 4.

Entretanto, vale ressaltar que, avaliando-se os resultados anatômicos pós-operatórios pela padronização de terminologia para pesquisadores de desordens do assoalho pélvico ${ }^{15}$, a maioria $(88,3 \%)$ foi considerada

Tabela 2 - Queixas referidas no pré-operatório, três e seis meses após a cirurgia.

\begin{tabular}{|c|c|c|c|c|}
\hline Queixas & $\begin{array}{c}\text { Pré-operutório } \\
\text { n (\%) }\end{array}$ & $\begin{array}{c}3 \text { meses } \\
n(\%)\end{array}$ & $\begin{array}{l}6 \text { meses } \\
n(\%)\end{array}$ & $\underset{\text { (início/fim) }}{\mathbf{p}}$ \\
\hline Dispareunia & $11(25,6)$ & $13(38,2)$ & $7(17,1)$ & \\
\hline Incômodo & $12(27,9)$ & - & - & \\
\hline Embaraço & $9(20,9)$ & - & - & $<0,001^{*}$ \\
\hline Medo & $1(2,3)$ & - & - & \\
\hline Sem queixas & $10(23,3)$ & $21(61,8)$ & $34(82,9)$ & \\
\hline Total & $43(100,0)$ & $34(100)$ & $41(100,0)$ & \\
\hline
\end{tabular}

"Valor do p obtido pelo teste de simetria de Bowker. 
Tabela 3 - Variação dos escores de desejo, excitação e satisfação no pré-operatório, três e seis meses após a cirurgia.

\begin{tabular}{|c|c|c|c|c|c|c|c|c|c|}
\hline \multirow{2}{*}{$\begin{array}{l}\text { Variável } \\
\text { Período }\end{array}$} & \multicolumn{3}{|c|}{ Desejo } & \multicolumn{3}{|c|}{ Excitação } & \multicolumn{3}{|c|}{ Satisfação } \\
\hline & Pré & $\begin{array}{c}3 \\
\text { meses }\end{array}$ & $\begin{array}{c}6 \\
\text { meses }\end{array}$ & Pré & $\begin{array}{c}3 \\
\text { meses }\end{array}$ & $\begin{array}{c}6 \\
\text { meses }\end{array}$ & Pré & $\begin{array}{c}3 \\
\text { meses }\end{array}$ & $\begin{array}{c}6 \\
\text { meses }\end{array}$ \\
\hline Média & 4,9 & 6,2 & 6,2 & 6,0 & 7,2 & 7,3 & 5,2 & 7,4 & 7,0 \\
\hline Desvio padrão & 2,9 & 2,5 & 2,7 & 2,3 & 2,5 & 1,8 & 2,7 & 2,1 & 2,5 \\
\hline Mínimo & 0 & 0 & 0 & 0 & 0 & 4 & 0 & 1 & 0 \\
\hline Máximo & 10 & 10 & 10 & 10 & 10 & 10 & 10 & 10 & 10 \\
\hline$p$ & \multicolumn{3}{|c|}{$0,001^{*}$} & \multicolumn{3}{|c|}{$<0,001^{*}$} & \multicolumn{3}{|c|}{$<0,001^{*}$} \\
\hline
\end{tabular}

"Valor do p obtido pelo teste de Wilcoxon.

Tabela 4 - Estádio clínico no pré-operatório, três e seis meses após a cirurgia.

\begin{tabular}{lcccc}
\hline Estádio & $\begin{array}{c}\text { Pré- } \\
\text { operatório } \\
\text { n (\%) }\end{array}$ & $\begin{array}{c}3 \text { meses } \\
\text { n }(\%)\end{array}$ & $\begin{array}{c}\text { 6 meses } \\
\text { n (\%) }\end{array}$ & p (início/fim) \\
\hline 0 & - & $4(9,3)$ & $2(4,7)$ & \\
I & - & $6(14,0)$ & $3(7,0)$ & \\
II & $27(62,8)$ & $32(74,4)$ & $37(86,0)$ & $<0,001^{*}$ \\
III & $10(23,2)$ & $1(2,3)$ & $1(2,3)$ & \\
IV & $6(14,0)$ & - & - & \\
Total & $43(100)$ & $43(100)$ & $43(100)$ & \\
\hline
\end{tabular}

*Valor do p obtido pelo teste de simetria de Bowker.

Tabela 5 - Estádio clínico seis meses após a cirurgia versus impacto da cirurgia na função sexual feminina.

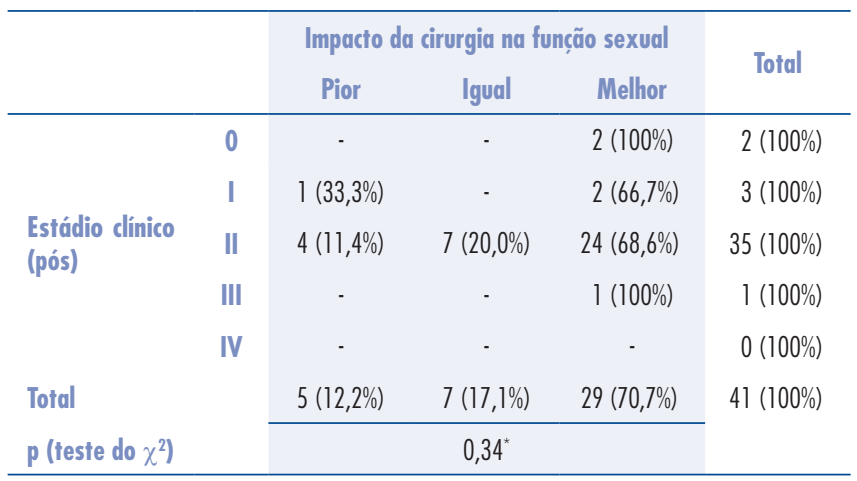

"Valor do p obtido pelo teste do $\chi^{2}$.

insatisfatória, principalmente em razão de recidiva da procidência de parede vaginal anterior $(79,1 \%)$. Quando avaliamos por compartimentos, a cúpula apresentou cura em $90,7 \%$ e melhora em $9,3 \%$ e a parede vaginal posterior, cura em $60,4 \%$ e melhora em $14 \%$.

Não houve associação significativa $(\mathrm{p}=0,34)$ entre a melhora da função sexual e a melhora do estádio clínico pós-cirúrgico (Tabela 5). Também não houve correlação entre a melhora da vida sexual pós-operatória e as mudanças nas variáveis "hiato genital" $(p=0,79)$ e "comprimento total da vagina" $(\mathrm{p}=0,14)$.

\section{Discussão}

Projeta-se que, nos próximos 30 anos, haja duplicação do número de pacientes que buscam tratamento por desordens do assoalho pélvico, em virtude do estilo de vida cada vez mais ativo das mulheres de terceira idade ${ }^{16}$.

As cirurgias para correção de distopia genital podem ser benéficas por abolirem os sintomas causados pela afecção, bem como pela reconstituição $\mathrm{da}$ anatomia ${ }^{17}$, mas tais procedimentos podem, em contrapartida, desencadear disfunção sexual por causa orgânica (dano neural, vascular, fibrose ou estenose) ou emocional (medo) ${ }^{18}$.

Deterioração da função sexual pós-cirurgia para correção de distopia e/ou incontinência urinária foi observada por estudo prospectivo, com redução do escore total de sexualidade aferido pelo instrumento de $\mathrm{McCoy}$ em $74 \%$ dos casos. Os autores atribuem a redução do escore à redução da elasticidade vaginal e a danos na inervação perineal, resultando em distúrbios na ereção reflexa, bem como redução do fluxo sangüíneo para os tecidos eréteis vaginais ${ }^{13}$.

Um artigo de revisão confirma a existência de impacto negativo das cirurgias de reconstrução do assoalho pélvico na função sexual feminina, particularmente quando se associa a plicatura dos músculos levantadores do ânus ${ }^{19}$. No presente estudo, no entanto, detectamos melhora significativa na qualificação da vida sexual e nos escores obtidos com as escalas analógicas de desejo, excitação e satisfação, apesar de não ter sido observada alteração significativa na freqüência de intercurso nem na freqüência de obtenção de orgasmos. Isto também foi demonstrado por estudo com metodologia similar, utilizando questionário validado, o índice de função 
sexual feminina, que detectou melhores escores pósoperatórios $(15,9 \pm 10,7 \text { para } 21,9 \pm 11,1)^{20}$.

Impacto positivo também foi demonstrado por estudo prospectivo multicêntrico com uso do questionário de avaliação sexual, prolapso genital e incontinência urinária (PSIQ), que detectou melhora dos escores de função sexual (89 para 95, $\mathrm{p}<0,001)^{21}$.

A ocorrência de dispareunia após colporrafia posterior é referida por alguns autores ${ }^{19,22}$. Estudo prospectivo multicêntrico utilizando o PSIQ em pacientes que se submeteram a cirurgias de reconstrução do assoalho pélvico comparou pacientes que se submeteram a reparo posterior com aquelas sem tal reparo. Os escores do PSIQ melhoraram no pós-operatório em ambos os grupos, apesar de a dispareunia ter sido mais freqüiente no grupo com reparo posterior $(28 \% \text { versus } 57 \% ; \mathrm{p}=0,002)^{23}$.

Neste estudo, houve melhora significativa da dispareunia, apesar de ter sido realizada perineoplastia com plicatura dos músculos levantadores do ânus em $84 \%$ das pacientes. Incômodo, embaraço e medo, referidos no pré-operatório, não foram demonstrados após a cirurgia. Talvez, a melhora significativa de tais queixas, associada à melhora da auto-estima por recuperação da auto-imagem, expliquem os nossos resultados satisfatórios.

Além disso, as pacientes eram cuidadosamente orientadas sobre a possível ocorrência de discreto incômodo nos primeiros intercursos, em geral, autolimitado, bem como sobre a impossibilidade de a atividade sexual danificar a cirurgia quando praticada após liberação médica. Acreditamos que tais informações também tenham contribuído para o impacto positivo $(70,7 \%$ de melhora) das cirurgias na função sexual de nossas pacientes, por abolirem o medo e a insegurança que podem surgir após a cirurgia.

Não houve associação significativa entre a melhora da função sexual e a melhora do estádio clínico pósoperatório de forma geral, nem quando se avaliou, em particular, o comprimento total da vagina e o hiato genital, o que também foi demonstrado por outros autores, que não detectaram correlação entre mudanças no comprimento total da vagina e seu calibre e alterações na função sexual ${ }^{10}$.

A padronização de terminologia para pesquisadores de desordens do assoalho pélvico define cura pós-operatória como estádio 0 ou ausência de prolapso. Resultado anatômico satisfatório (melhora) é considerado como estádio I e resultado anatômico insatisfatório (persistência ou recorrência) após a intervenção é definido como estádio II ou mais, ou inalteração ou piora em relação ao pré-operatório ${ }^{15}$.

Apesar da melhora significativa entre os estádios préoperatório e seis meses após a cirurgia de nossa casuística, segundo tal padronização, a maioria $(88,3 \%)$ dos resultados anatômicos pós-cirúrgicos deste estudo foi insatisfatória. Esta padronização não considera que a mudança de um estádio IV para II seja satisfatória, apesar de tal alteração ser significativa estatística e clinicamente.

Os autores reconhecem que muitas mulheres classificadas como apresentando falhas anatômicas após cirurgias encontram-se satisfeitas com o resultado pós-operatório e que a definição de prolapso deveria incluir a presença e a severidade dos sintomas. Assim, a distinção entre falha e cura pós-intervenção deveria incluir a resolução ou a persistência de tais sintomas ${ }^{15}$. Concordamos plenamente com essas considerações, uma vez que, apesar dos resultados anatômicos insatisfatórios encontrados, apenas uma paciente permaneceu sintomática após a cirurgia (estádio IIIc), tendo se submetido a nova correção da distopia com tela de polipropileno $\left(\right.$ Apogee ${ }^{\circledR}$ ) no quarto mês pós-operatório.

Nosso estudo tem algumas limitações: sua natureza observacional impossibilita inferências de causa e efeito. No entanto, testar hipóteses específicas não foi o objetivo inicial, e sim, avaliar as repercussões das cirurgias sobre a função sexual feminina. As conclusões são reforçadas pelo uso dos mesmos parâmetros de avaliação no pré e no pós-operatório, de modo que cada mulher funciona como seu próprio controle no tocante à função sexual. Importa, ainda, o fato de que não houve troca de parceiro em nenhum caso, o que poderia funcionar como viés.

A pequena casuística se deve à dificuldade de seleção de pacientes que satisfizessem os critérios de inclusão, uma vez que a distopia, em geral, se apresenta em pacientes mais idosas e, quanto maior a faixa etária, mais freqüente a falta de atividade sexual por problemas de saúde do parceiro, viuvez ou falta de desejo da mulher, conforme demonstra a literatura, que refere que mulheres com idade entre 46 e 50 anos são sexualmente ativas em $86 \%$ dos casos, entre 56 e 60 anos em $58 \%$ e entre 61 e 65 anos em $39 \%$ apenas $^{24}$. A média de idade das pacientes deste estudo foi de 52,9 anos. Ressalta-se, no entanto, a excelente adesão ao seguimento. Todas as pacientes retornaram em três e em seis meses, o que não foi demonstrado em outros estudos, com adesão entre 51 e $89 \%{ }^{11,13,20}$.

Cumpre ainda mencionar a dificuldade em avaliar a função sexual, uma vez que a percepção de satisfação sexual depende de vários fatores. Bem-estar emocional, intimidade com o parceiro, qualidade de vida e saúde física são alguns deles ${ }^{6}$. Alguns métodos de avaliação da função sexual são referidos na literatura. A fotopletismografia, a avaliação da temperatura labial, a dopplervelocimetria e a ressonância magnética nuclear podem fornecer medidas 
objetivas da resposta sexual. No entanto, pela natureza invasiva, pouca praticidade e altos custos de tais métodos, têm sido utilizadas, com mais freqüência, as medidas indiretas, por meio de questionários ${ }^{25}$.

A falta de um questionário condição-específico validado no Brasil no início deste estudo foi outra limitação importante. Desse modo, utilizou-se o questionário de Abdo et al. ${ }^{14}$, elaborado para avaliação do perfil sexual do brasileiro, que não possui questões especificamente desenhadas para elucidar a complexa interação entre as desordens do assoalho pélvico e a função sexual.
Estimulamos, então, a elaboração de um questionário condição-específico validado no Brasil. O mesmo deve ser, idealmente, curto, multidimensional, de fácil compreensão e com boa validade e confiabilidade, para que seja aplicado largamente: não apenas na execução de novos estudos epidemiológicos, mas também na prática clínica, uma vez que, mais importante que os resultados anatômicos pós-operatórios, seria a avaliação da satisfação das pacientes com o procedimento do ponto de vista funcional e, em particular, da função sexual, importante constituinte da saúde da mulher.

\section{Referências}

1. Laumann EO, Gagnon JH, Michael RT, Michaels S. The social organization of sexuality: sexual practices in the United States. Chicago: University of Chicago Press; 1994.

2. Abdo C. Estudo da Vida Sexual do Brasileiro. São Paulo: Bregantini; 2004.

3. Laumann EO, Paik A, Rosen RC. Sexual dysfunction in the United States: prevalence and predictors. JAMA. 1999;281(6):537-44.

4. Pauls RN, Kleeman SD, Segal JL, Silva WA, Goldenhar LM, Karram MM. Practice patterns of physician members of the American Urogynecologic Society regarding female sexual dysfunction: results of a national survey. Int Urogynecol J Pelvic Floor Dysfunct. 2005; 16(6):460-7.

5. Burrows L, Meyn LA, Walters MD, Weber AM. Pelvic symptoms in women with pelvic organ prolapse. Obstet Gynecol. 2004;104(5 Pt 1):982-8.

6. Barber MD, Visco AG, Wyman JF, Fantl JA, Bump RC; Continence Program for Women Research Group. Sexual function in women with urinary incontinence and pelvic organ prolapse. Obstet Gynecol. 2002;99(2):281-9.

7. Novi JM, Jeronis S, Morgan MA, Arya LA. Sexual function in women with pelvic organ prolapse compared to women without pelvic organ prolapse. J Urol. 2005;173(5):1669-72.

8. Rosenbaum TY. Pelvic floor involvement in male and female sexual dysfunction and the role of pelvic floor rehabilitation in treatment: a literature review. J Sex Med. 2007;4(1):4-13.

9. Haase P, Skibsted L. Influence of operations for stress incontinence and/or genital descensus on sexual life. Acta Obstet Gynecol Scand. 1988;67(7):659-61.

10. Weber AM, Walters MD, Piedmonte MR. Sexual function and vaginal anatomy in women before and after surgery for pelvic organ prolapse and urinary incontinence. Am J Obstet Gynecol. 2000;182(6): 1610-5.

11. Rogers RG, Kammerer-Doak D, Darrow A, Murray K, Olsen A, Barber $M$, et al. Sexual function after surgery for stress urinary incontinence and/or pelvic organ prolapse: a multicenter prospective study. Am J Obstet Gynecol. 2004;191(1):206-10.

12. Bump RC, Mattiasson A, Bo K, Brubaker LP, De Lancey JO, Klarskov $P$, et al. The standardization of terminology of female pelvic organ prolapse and pelvic floor dysfunction. Am J Obstet Gynecol. 1996;175(1):10-7.
13. Helstrom L, Nilsson B. Impact of vaginal surgery on sexuality and quality of life in women with urinary incontinence or genital descensus. Acta Obstet Gynecol Scand. 2005;84(1):79-84.

14. Abdo CHN, Moreira Junior ED, Fittipaldi JAS. Estudo do comportamento sexual no Brasil - ECOS. RBM Rev Bras Med. 2000;57(11):1329-35.

15. Weber AM, Abrams P, Brubaker L, Cundiff G, Davis G, Dmochowski $R R$, et al. The standardization of terminology for researchers in female pelvic floor disorders. Int Urogynecol J Pelvic Floor Dysfunct. $2001 ; 12(3): 178-86$

16. Drutz HP, Alarab M. Pelvic organ prolapse: demographics and future growth prospects. Int Urogynecol J Pelvic Floor Dysfunct. 2006; 17 Suppl 1:S6-9.

17. Pauls RN, Berman JR. Impact of pelvic floor disorders and prolapse on female sexual function and response. Urol Clin North Am. 2002;29(3):677-83

18. Tunuguntla HS, Gousse AE. Female sexual dysfunction following vaginal surgery: a review. J Urol. 2006;17(2):439-46.

19. Ghielmetti T, Kuhn P, Dreher EF, Kuhn A. Gynaecological operations: do they improve sexual life? Eur J Obstet Gynecol Reprod Biol. 2006; 129(2):104-10.

20. Azar M, Noohi S, Radfar S, Radfar MH. Sexual function in women after surgery for pelvic organ prolapse. Int Urogynecol J Pelvic Floor Dysfunct. 2007 jun 15; [Epub ahead of print].

21. Rogers RG, Kammerer-Doak D, Darrow A, Murray K, Qualls $C$, Olsen $A$, et al. Does sexual function change after surgery for stress urinary incontinence and/or pelvic organ prolapse? A multicenter prospective study. Am J Obstet Gynecol. 2006; 195(5):e 1-4.

22. Kahn MA, Stanton SL. Posterior colporrhaphy: its effects on bowel and sexual function. Br J Obstet Gynaecol. 1997;104(1):82-6.

23. Komesu YM, Rogers RG, Kammerer-Doak D, Barber MD, Olsen AL. Posterior repair and sexual function. Am J Obstet Gynecol. 2007; 197(1):101.e1-6.

24. Pfeiffer E, Verwoerdt A, Davis GC. Sexual behaviour in middle life. Am J Psychiatry. 1972;128(1):1262-7.

25. Rosen RC. Assessment of female sexual dysfunction: review of validated methods. Fertil Steril. 2002;77 Suppl 4:S89-93. 